\title{
Internet of Things (IoT) Platform for Structure Health Monitoring
}

\author{
Ahmed Abdelgawad and Kumar Yelamarthi \\ School of Engineering \& Technology, Central Michigan University, ET100, Mount Pleasant, MI 48859, USA \\ Correspondence should be addressed to Ahmed Abdelgawad; abdella@cmich.edu
}

Received 20 July 2016; Revised 6 October 2016; Accepted 19 October 2016; Published 16 January 2017

Academic Editor: Dajana Cassioli

Copyright (c) 2017 Ahmed Abdelgawad and Kumar Yelamarthi. This is an open access article distributed under the Creative Commons Attribution License, which permits unrestricted use, distribution, and reproduction in any medium, provided the original work is properly cited.

\begin{abstract}
Increase in the demand for reliable structural health information led to the development of Structural Health Monitoring (SHM). Prediction of upcoming accidents and estimation of useful life span of a structure are facilitated through SHM. While data sensing is the core of any SHM, tracking the data anytime anywhere is a prevailing challenge. With the advancement in information technology, the concept of Internet of Things (IoT) has made it possible to integrate SHM with Internet to track data anytime anywhere. In this paper, a SHM platform embedded with IoT is proposed to detect the size and location of damage in structures. The proposed platform consists of a Wi-Fi module, a Raspberry Pi, an Analog to Digital Converter (ADC), a Digital to Analog Converter (DAC), a buffer, and piezoelectric (PZT) sensors. The piezoelectric sensors are mounted as a pair in the structure. Data collected from the piezoelectric sensors will be used to detect the size and location of damage using a proposed mathematical model. Implemented on a Raspberry $\mathrm{Pi}$, the proposed mathematical model will estimate the size and location of structural damage, if any, and upload the data to Internet. This data will be stored and can be checked remotely from any mobile device. The system has been validated using a real test bed in the lab.
\end{abstract}

\section{Introduction}

SHM is a vital tool to improve the safety and maintainability of critical structures such as bridges and buildings. SHM provides real time and accurate information about the structural health condition. It is a process of nondestructive evaluations to detect location and extent of damage, calculate the remaining life, and predict upcoming accident. SHM has become challenging task with the increase in development and construction of structures along with the complexities involved in them. The demand for SHM has also increased due to increase in the necessity to ensure safety of the structures as well as the human lives associated with it. SHM is capable of detecting the damage early and make it feasible to take action before any loss occurs [1]. Diverse theories have been proposed and implemented to meet distinct requirements of structures. Integration of the diverse theories has helped not only to improve the efficiency and performance of the SHM systems but also to reduce the computational time and costs [2]. In order to share data and ensure reliability, SHM systems use networks [3].

We begin to coexist and interact with smart interconnected devices that are known as the IoT. The IoT brings new opportunities for our society. With the maturity of the IoT, one of the recent challenges in the structural engineering community is development of the IoT SHM systems that can provide a promising solution for rapid, accurate, and low-cost SHM systems. Moreover, the combination of SHM, cloud computing, and the IoT enabled ubiquitous services and powerful processing of sensing data streams beyond the capability of traditional SHM system. Cloud platform allows the SHM data to be stored and used intelligently for smart monitoring and actuation with smart devices. In this paper, a complete SHM platform embedded with IoT is proposed to detect the size and location of damage in structures. 


\section{Previous Work}

SHM process incorporates sensors, signal processing, and hardware implementation. Many sensors, for example, accelerometer, ultrasonic, laser, vibration, camera, fiber optical, and piezoelectric have been used. Terrestrial Laser Scanning (TLS) technique uses laser sensor to get the threedimensional coordinates of the target structure and monitor its health. Laser Doppler velocity meter is also used in SHM using noncontact and low frequency lamb-wave detection [4]. The detection of the structural damage can be made more robust by using a triaxis, multiposition scanning laser vibrometer [5]. A displacement measurement model has been incorporated with it to reduce the error of TLS by using linear variable displacement transducer (LVDT), electric strain gages, and long gage fiber optic sensor. To monitor the structural health, a 3D finite element model takes the periodic measurement of the deformation structure and performs inverse structural analysis with the measured $3 \mathrm{D}$ displacements [6].

Image processing technique involved in multipath ultrasonic guided wave imaging is used for complex structures, inhomogeneous and anisotropic materials. This technique gives an improved image quality using fewer sensors. It takes maximum advantage of using a large number of echoes and reverberations to perform localization and damage detection. This system helps not only to increase the performance but also to reduce complexity and cost [7, 8]. Vibrational sensors and video cameras used together in Wireless Sensor Networks (WSN) send distributed data interpretation to detect the local data trends like normal vibrations, abnormal vibrations, and structural tilts. The video camera is prompted to zoom/tilt into the local area for monitoring in case of critical events. This video camera data is synchronized with the sensor data $[9,10]$. The self-diagnosis system helps to find out the failed piezoelectric sensors and reconfigure the network with existing sensors in large SHM systems [11, 12]. Piezoelectric wafer active sensors (PWAS) are used in a variety of damage detection methods $[13,14]$. PWAS are nonresonant devices and can be used in various guided wave modes [15]. Piezoelectric sensors have extended its groves in multiple fields like aircraft, aerospace health conditioning, metallic plate stiffener disbanding, and structural health of wind turbines [16-19]. PWAS are very useful in large class of SHM systems like guided wave ultrasonic and electromechanical and passive detection [20,21].

Fiber optical sensors are used in Fiber Bragg Grating (FBG) to detect the dynamic loads on bridge decks [22]. Vibration based SHM systems have become an area of focus in recent studies, as it is used to detect damage that cannot be visually detected and damage hidden within the internal areas of the structure. The vibration of the structure changes along with the stiffness of the body when it is damaged and this can be detected by using vibrational SHM [23]. The usage of Wireless Sensor Networks (WSN) in SHM systems has increased in recent times due to its low installation and maintenance costs [24]. Compared to other data acquisition systems, WSN has various benefits such as low maintenance cost, flexibility, and ease of deployment [25].
Signal processing is the key component of any SHM. The data obtained through data acquisition systems are not accurate as there are vibrational and environmental effects which influence the output data. The errors in the output data can be removed by using Principal component analysis (PCA) and Hilbert-Huang Transform (HHT) with EMD for data processing and analyzing data to detect structural health problems [26, 27]. The guided wave SHM system with digital signal processing module executes excitation of signal sensing and data processing. The instantaneous baseline damage detection based on Wavelet Transformation (WT) and cross correlation (CC) analysis is carried out by the DSP module of low-power piezoelectric guided waves system [28]. To achieve a high accuracy and resolution, Gao et al. proposed a system which synchronizes the time to ensure data acquisition and propagation delay calculation. A TMS320F28335 digital signal processor (DSP) and an improved IEEE 802.15.4 wireless data transducer are used [29]. The Wavelet Spectral Finite Element (WSFE) method is very efficient for wave motion analysis and best suitable for inverse problem solving but will have "wraparound" problem. Samaratunga has proposed WSFE model which eliminates the wraparound problem and is best suitable for 2D finite structures with transverse cracks [30]. A wavelet based approach is also helpful in finding the damage from wavelet decomposition of response data. Location of the damage can be detected by patterns in the spatial distribution of spikes [31]. Even with the existence of all these systems, we lag to integrate the data with the Internet. Tracking of data is also as important as sensing the health of the structure.

Hardware implementation is necessary for the practical implementation of SHM. Different types of hardware implementation techniques are used by many researchers. Park et al. proposed an online instantaneous baseline structural damage detection technique using a low-cost and low-power piezoelectric guided waves system. A TMS320F2812 digital signal processing (DSP) module was employed for signal generation/excitation, sensing, and data processing. The DSP applied an instantaneous baseline damage identification algorithm based on WT and CC analysis [28]. Traditional wired SHM system requires long deployment time and a significant cost for wire installation. Last decade, a large number of researchers have been focused on using Wireless Sensor Network (WSN). Gao et al. presented a real-time wireless piezoelectric sensor platform for distributed large-scale SHM with high sampling rate up to $12.5 \mathrm{Msps}$ and distributed lambwave data processing. A set of PZT sensors were deployed at the surface of the structure. A lamb wave was excited and its propagation within the structure was inspected to identify damage. The developed wireless node platform features a DSP of TMS320F28335 and an improved IEEE 802.15.4 wireless data transducer RF233 with up to $2 \mathrm{Mbps}$ data rate [29]. There are many other attempts to implement SHM using Wireless Sensor Network (WSN) [32-35].

Lazo et al. proposed a solution for communicating devices that monitor the health of a bridge. The proposed solution is based on 6LoWPAN, a standard based on the IPv6 protocol over low-power and lossy networks, to support the 


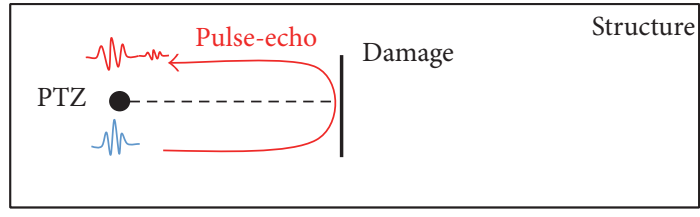

(a)

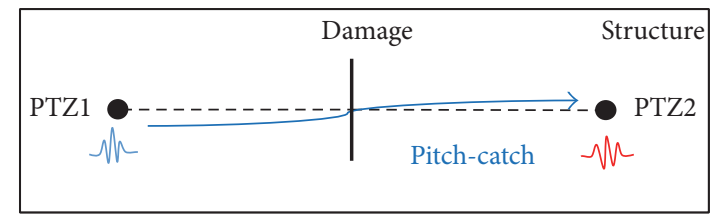

(b)

FIGURE 1: Guided waves damage detection techniques: (a) pulse-echo and (b) pitch-catch.

SHM IoT system [36]. Zhang et al. proposed an environmental effect removal based SHM scheme in an IoT system. They employed principal component analysis (PCA) to eliminate environment effects from sensor data. After environmental effects removal, Hilbert-Huang transformation (HHT) was used for structural health analysis and monitoring [26]. Both $[26,36]$ attempts are simulation-based research. Yet, there is no real implementation of SHM in the IoT. This paper proposes a complete real-time IoT platform for SHM.

This paper is organized as follows. Section 2 presents the design for SHM system using piezoelectric sensor, followed by the proposed mathematical model in Section 3 and damage detection using a cross correlation process in Section 4. In Section 5, the results of the system validation are provided. Finally, Section 6 concludes this paper.

\section{SHM Damage Detection Techniques}

Damage detection in SHM systems is classified into two types: local-based and global-based damage detection [37]. Localbased damage detection is used for screening structures and global-based damage detection is used for vibrational characteristics. Various sensors are used to detect the size and location of the damage in SHM systems [38]. Sensors like piezoelectric, optical fiber, ultrasonic, laser, image detection, and vibrational sensors are commonly used because of their leverage over other sensors.

Piezoelectric sensors are the most commonly used sensors; they can transmit and receive guided waves such as lamb waves in solids that can be used for damage detection. These lamb waves are much more cost effective as well as reliable as they are sensitive to change in structures. To identify the damage, the difference signal is acquired and compared with a damage-free signal. For structural damage localization, irrespective of the geometrical or imaging method used, the key to this process is the acquired time of flight and amplitude of the response to the signal. These factors directly determine the precision of damage localization. The time of flight is directly dependent on the properties of the material such as modulus of elasticity and modulus of rigidity. Using piezoelectric sensors, there are two basic active sensing techniques used to detect damage in structures, namely, pitch-catch and pulse-echo. In the pitch-catch technique, two PZT sensors are used: one as transmitter that located before the damage and the other as a receiver that located after the damage. The first PZT sends a signal, and the received signal by the second PZT is then used to determine the location and/or the extent of the damage [11]. On the other

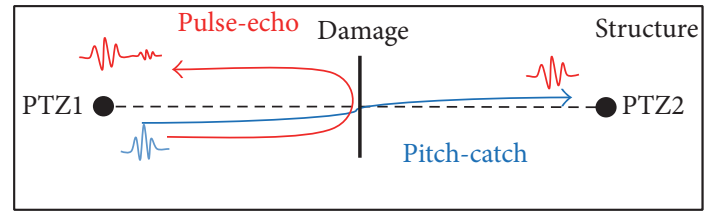

FIGURE 2: The proposed SHM technique.

hand, the pulse-echo technique relies on the reflected wave from the damage, and one PTZ transducer is used for both transmitting and receiving the signal. Figure 1 shows both techniques.

\section{Proposed SHM Technique}

In this paper, a combination of pulse-echo and pitch-catch techniques is proposed as shown in Figure 2. Pitch-catch technique is used to determine presence of damage. If any damage is found, the pulse-echo technique will be used to determine location of the damage. This method uses two PZT sensors. The first transducer (PZT1) excites the signal towards the second transducer (PZT2) and picks up any waves that have been reflected back from damage that could not reach the second transducer, which uses the pulse-echo technique. The second, PTZ2, will receive wave feedback in a pitchcatch manner. The proposed technique is good for any metal structure, for example, aluminum and steel.

In order to detect if the structure is healthy or not, a cross correlation (CC) algorithm is used to check the similarity between excitation signal, $E$, at PTZ1 and received signal, $R$, at PTZ2. The CC value is used as a linear damage index and is defined as

$$
\mathrm{CC}=\frac{(1 / N) \sum_{i=1}^{N}\left(E_{i}-\bar{E}\right)\left(R_{i}-\bar{R}\right)}{\sigma_{E} \sigma_{R}},
$$

where $\bar{E}$ and $\bar{R}$ are the mean values of the two sets of signal and $\sigma_{E}$ and $\sigma_{R}$ are standard deviations of the signature signal sets $E$ and $R$, respectively. If the two signals are similar, CC $\approx 1$, which mean that the structure is healthy; otherwise, the structure has damage. In order to determine the size and location of the damage, a mathematical model is proposed based on the dimensional diagram shown in Figure 3. 


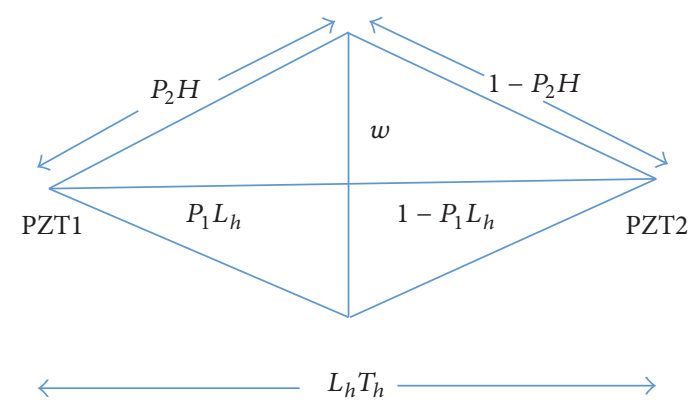

FIGURE 3: Dimensional diagram for the proposed SHM technique.

First, the speed of the wave must be calculated using a healthy model as in

$$
\frac{L_{h}}{T_{h}}=W_{s},
$$

where $L_{h}$ is the length from exciter to sensor and $T_{h}$ is the time between peak-peak. In order to find the damage location, $L_{c}$, we are using the following:

$$
\frac{T_{c}}{2} W_{s}=L_{c}
$$

where $T_{c}$ is the time it takes for a wave to travel to the damage and reflect back. After calculating the position, the next step would be determining the damage width. First, the proportion of the damage position to the total length, $P_{1}$, could be determined by

$$
\frac{L_{c}}{L_{h}}=P_{1} .
$$

Due to the damage being present, the wave takes longer to travel from exciter to sensor, which implies that the wave travels a further distance. This distance, $H$, can be determined by

$$
T_{t} W_{s}=H
$$

where $T_{t}$ is the total time the wave takes to travel from exciter to the sensor with the presence of the damage. From these equations, a triangle can be made as seen in Figure 3. $L_{c}$ is the base and $P_{2} H$ is hypotenuse of this triangle. $P_{1}$ and $P_{2}$ are very close in value and only equal if $P_{1}$ is $1 / 2 . P_{2}$ can be determined using the following:

$$
\frac{\left(H^{2}-L_{c}^{2}+2 L_{c}^{2} P_{1}\right)}{2 H^{2}}=P_{2} .
$$

After $P_{2}$ is calculated, the width of the damage is determined from the exciter/sensor axis using the Pythagorean Theorem:

$$
\sqrt{\left(P_{2} H\right)^{2}-L_{c}^{2}}=W
$$

These equations are used if the damage has the same width above and below the axis. If the damage is not symmetrical, the two waves might reach the sensor at slightly different

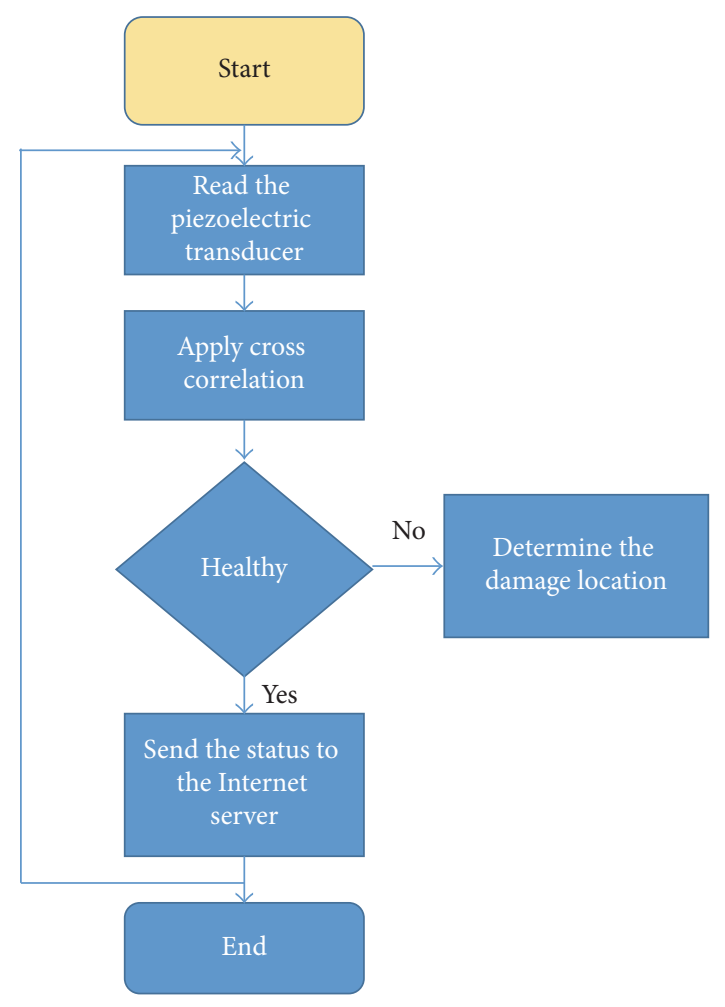

FIGURE 4: Proposed SHM flow chart.

times resulting in two $T_{t} \mathrm{~s}$, two $H \mathrm{~s}$, and two $P_{2}$ s to calculate the upper and lower part of the damage. From the above mathematical model, the damage location can be detected using (5) and the damage width can be determined using (7). One unique advantage of the proposed mathematical model is that it does not require high computational processing unit, allowing for implementation almost anywhere, anytime, and also easier integration with an IoT platform. Figure 4 shows the flow chart of the proposed SHM technique.

\section{Evaluation of the Proposed Mathematical Model}

In order to evaluate the proposed mathematical model, ABAQUS software [39] is used to simulate different scenarios for different damage location and width. The waves at both PZT sensors will be collected by Matlab [40], which will run the proposed mathematical model to determine the damage location and width. The output of the proposed model, damage location and width, will be compared with the actual values, as measured. A $40 \mathrm{~cm} \times 40 \mathrm{~cm}$ steel plate of $2 \mathrm{~mm}$ thickness was used for the simulations. Steel is one of the most widely used metals in solid structures in the world. Not only this, but steel is a brittle material that has the tendency to damage under stress rather than yield like ductile materials such as aluminum. Although it would be possible to use another metal as well, it was chosen due to its physical properties. Further, any metal can be used as long as the corresponding physical property inputs are changed within the system. Two sensors were used at very specific 


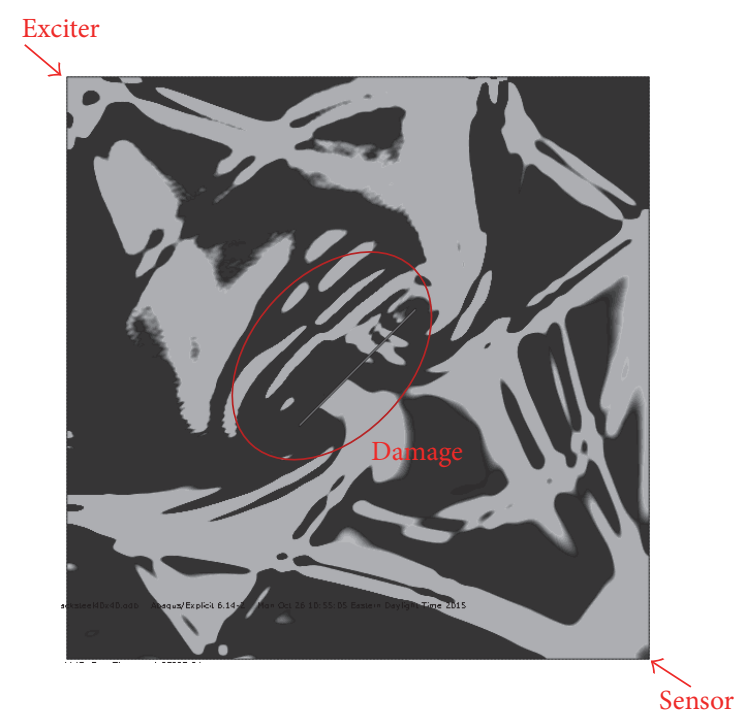

FIgURE 5: Simulation in ABAQUS with steel plate.

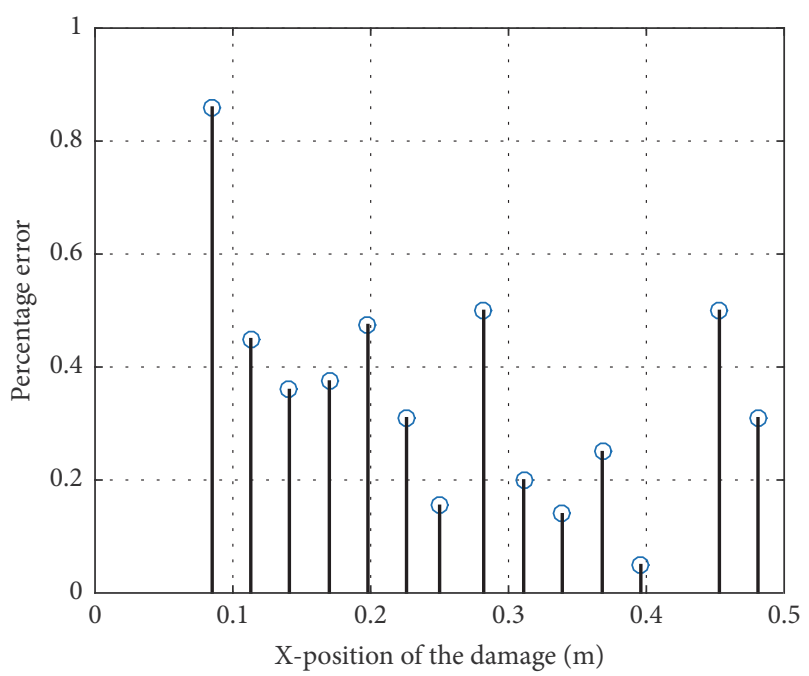

Figure 6: Percentage error of the calculated position of the damage.

locations, which are two opposing corners of the metal. This reduces the amount of unwanted wave reflections from the boundaries of the specimen, also known as noise. The exciter sends radial waves in all directions, so, by placing it on a corner, the reflections will essentially be sent in a single 90degree radius direction with no unwanted noise. A linear force at a frequency of $243 \mathrm{kHz}$ was used to generate the waves [41]. Figure 5 shows the simulation in ABAQUS with steel plate.

Damage was considered along the diagonal of this plate starting with a distance starts at $85 \mathrm{~mm}$ and ends with $481 \mathrm{~mm}$ from the exciter. Different simulation scenarios with different damage location along the plate's diagonal have been done. Figure 6 shows the percentage error between the actual damage location and the damage location calculated by the proposed technique. The results show that the proposed model determines the damage location precisely with a maximum of $0.9 \%$ error.

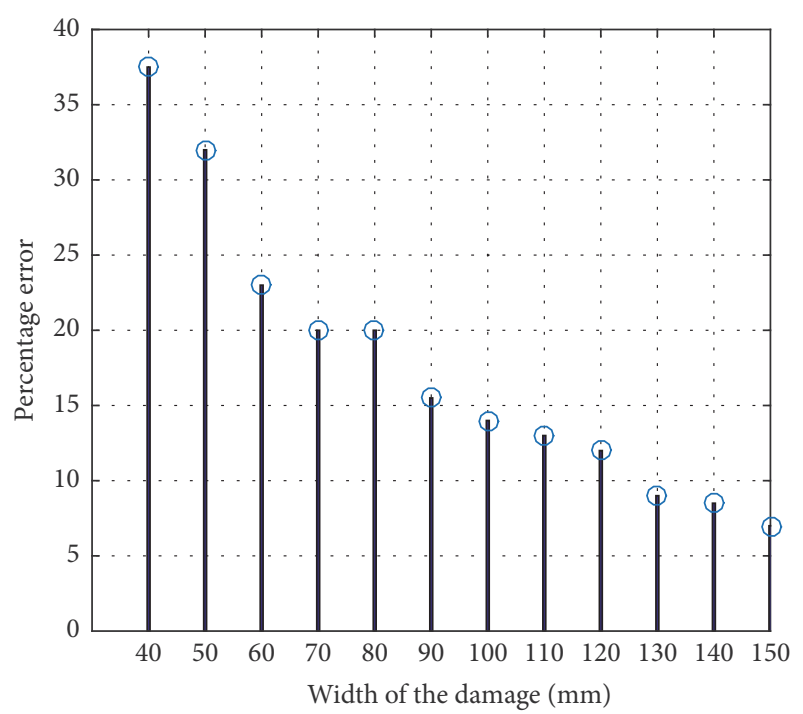

FIgURE 7: Percentage error of calculated damage width for changing width at the center of the steel sheet.

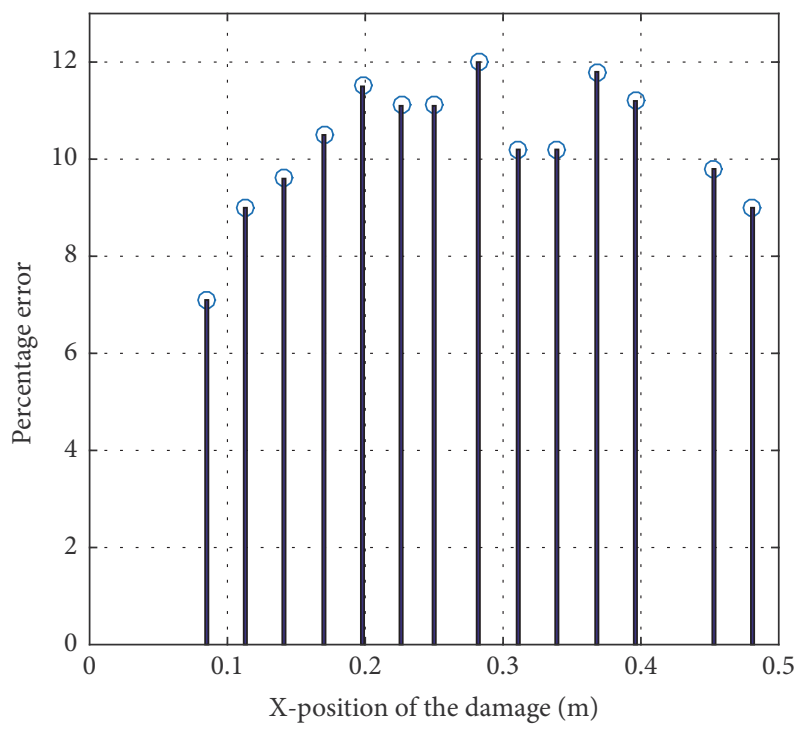

FIgURE 8: Percentage error of calculated damage width while the damage position is changing.

In order to evaluate the damage width calculation, different simulation scenarios for different damage width have been done. First, we considered damage located at center of the plate along with the diagonal. Second, the simulation was done for differed damage widths. Figure 7 shows the percentage error between the actual damage width and the damage width calculated by the proposed technique. The results show that the proposed technique was able to determine the damage width with an average of $17.5 \%$ error.

It can be noticed that the proposed technique's error increases when the damage width decreases. Thus, evaluating the proposed technique for a fixed damage width at different locations is very important. Figure 8 shows the percentage error between the actual damage width and the damage 


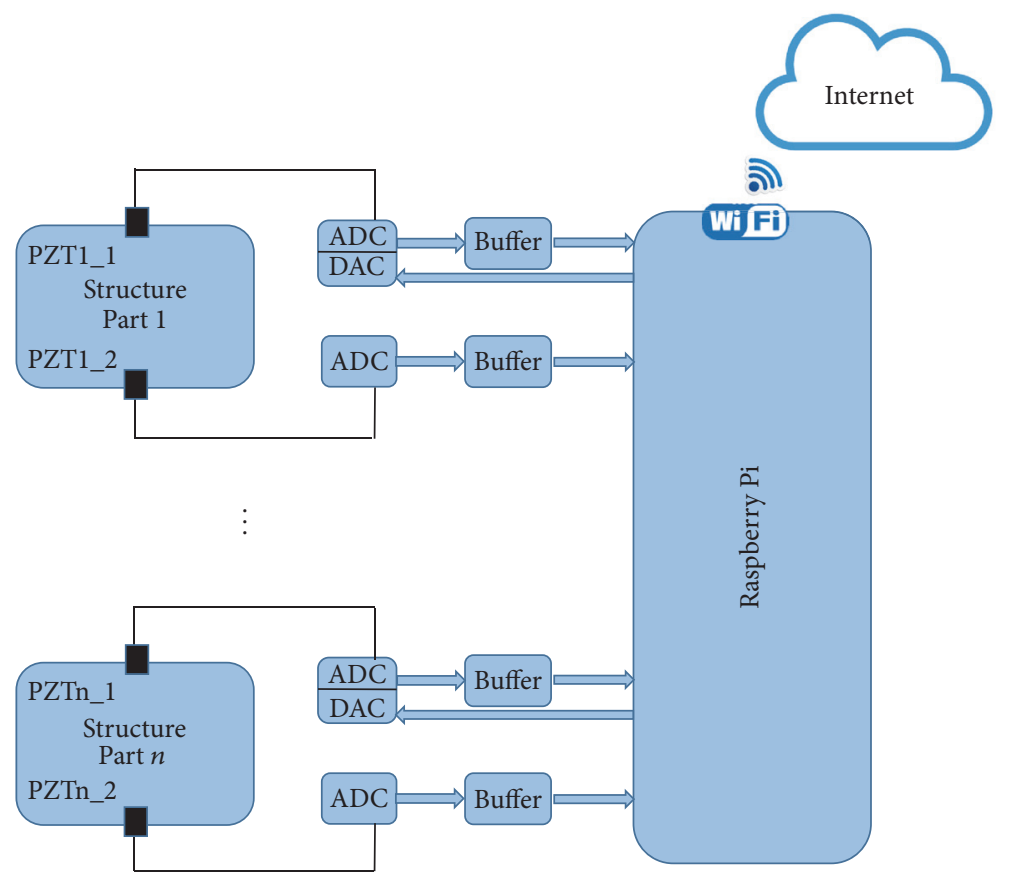

FIgURE 9: Proposed IoT platform for SHM.

width calculated by the proposed technique for a fixed width at different locations. The results show that the proposed technique is able to determine the damage width with an average of $10.4 \%$ error. Results show that the proposed mathematical model is accurate. Moreover, it does not need high computational processing unit. Thus, this mathematical model could be integrated with IoT platform.

\section{Proposed IoT Platform for SHM}

The proposed IoT platform consists of Wi-Fi module, Raspberry Pi, ADC, DAC, buffer, and PZT as shown in Figure 9. The two piezoelectric sensors are mounted on the structural and connected to a high speed ADC. In the real case implementation, we will deploy the sensors in a way to catch all the possible damage. A buffer is used as a level conversion and to protect the Raspberry Pi. The Raspberry $\mathrm{Pi}$ generated the excitation signal and the DAC converted it to analog. In addition, the Raspberry $\mathrm{Pi}$, using the proposed SHM technique, is used to detect if the structure has damage or not and the location of the damage if it is existing. Moreover, the Raspberry sends the structure health status to the Internet server. The data is stored on the Internet and can be monitored remotely from any mobile device. Moreover, the Internet server sends an alert if there is a damage in the structure.

6.1. Wi-Fi Module. Miniature Wi-Fi $(802.11 \mathrm{~b} / \mathrm{g} / \mathrm{n})$ Module is a USB module that has $2.4 \mathrm{GHz}$ ISM band. It has a data rate up to $150 \mathrm{Mbps}$ (downlink) and up to $150 \mathrm{Mbps}$ (uplink). It uses IEEE 802.11n (draft), IEEE 802.11g, and IEEE 802.11b standards. The Wi-Fi module is used to send the data to the cloud.

6.2. Raspberry $P i$ 2. The Raspberry Pi 2 is a single-board computer. It features a full Linux operating system with diverse programming and connectivity options. The onboard $900 \mathrm{MHz}$ quad-core ARM Cortex-A7 CPU allows for swift computation and analysis of data obtained from several nodes and transducers. The operating system of Raspberry $\mathrm{Pi}$ is Linux 3.18.5-v7+ and has 4 processors in one chip which is CPU ARMv7 Processor rev 5 (v7l). It has a RAM of $1 \mathrm{~GB}$ and maximum clock speed $900 \mathrm{MHz}$. Normally, current drawn by Raspberry Pi 2 is $200 \mathrm{~mA}$. The Raspberry Pi will be used to collect the structure health and push it to the cloud using WiFi module.

6.3. ADC. The CA3306 is a CMOS parallel ADC designed for applications demanding both low-power consumption and high speed digitization. It is a 6-bit 15 MSPS ADC with a parallel read out with single $5 \mathrm{~V}$ supply. The power consumption is as low as $15 \mathrm{~mW}$, depending upon the operating clock frequency. It may be directly retrofitted into CA3300 sockets, offering improved linearity at a lower reference voltage and high operating speed with a $5 \mathrm{~V}$ supply. The high conversion rate of this ADC is ideally suited for digitizing high speed signals in SHM application. If a higher resolution is needed, the overflow bit makes the connection of two or more CA3306s in series possible to increase the resolution. Also, two CA3306s may be used to produce a 7-bit high speed converter that doubles the conversion speed; this will increase the sampling rate from $15 \mathrm{MHz}$ to $30 \mathrm{MHz}$ [42]. 


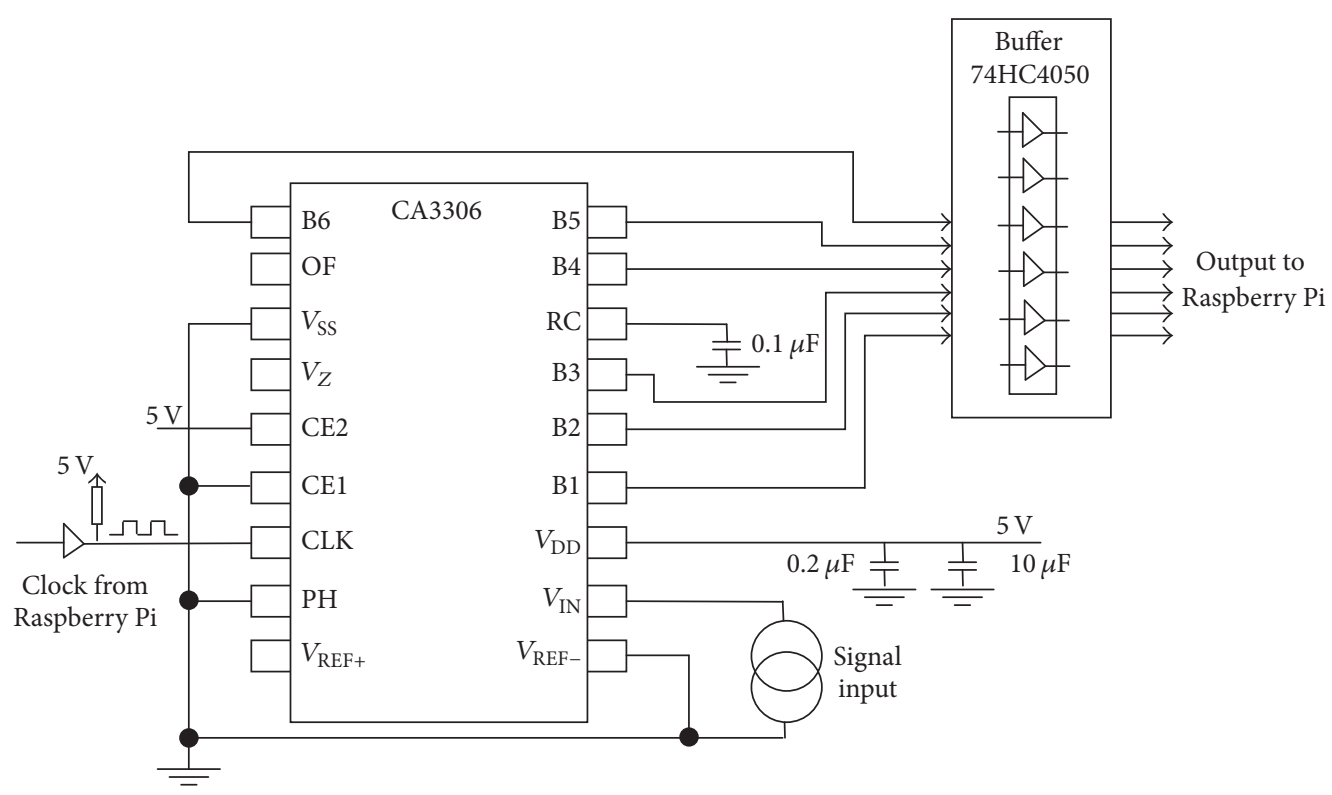

FIgURE 10: The circuit diagram of both the ADC and the buffer.

6.4. DAC. The MCP4725 is a low-power, high accuracy, single channel, 12-bit buffered voltage output DAC with nonvolatile memory (EEPROM). Its onboard precision output amplifier allows it to achieve rail-to-rail analog output swing. The MCP4725 is an ideal DAC device where design simplicity and small footprint are desired and for applications requiring the DAC device settings to be saved during power-off time [43].

6.5. Buffer. The $74 \mathrm{HC} 4050$ is a hex buffer with overvoltage tolerant inputs. Inputs are overvoltage tolerant up to $15 \mathrm{~V}$ which enables the device to be used in high-to-low level shifting applications [44].

6.6. Piezoelectric Sensors. The PZT transducer converts mechanical energy to electric signals or vice versa. They can work as an actuator to excite an elastic lamb wave based on the electrical signal applied to the PZT crystal. It can also be used as a transducer to transform the responding elastic lamb waves into an electrical signal. Two PTZs sensors are mounted on structure: PZT1 (excitation) will send the excitation signal and PZT2 (receiver) will receive the signal.

The CA3306 is operating at $5 \mathrm{~V}$, which means that we cannot connect it directly to the Raspberry Pi which operates at $3.3 \mathrm{~V}$. Accordingly, a level converter in between is needed. The simplest way to do level conversion is to use a buffer such as CMOS 74HC4050. As the buffer runs at $3.3 \mathrm{~V}$, so it is necessary to place a pull-up resistor to $5 \mathrm{~V}$ behind the clock buffer. Figure 10 shows the circuit diagram of both the ADC and the buffer.

\section{Test and Evaluation}

In order to evaluate the proposed platform, a healthy aluminum sheet and another sheet with damage were used. The

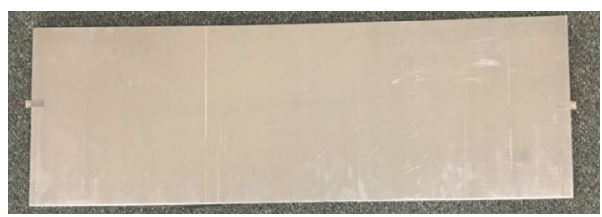

(a)

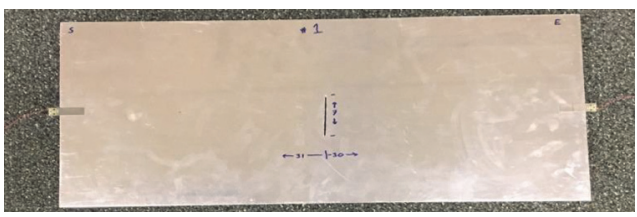

(b)

FIGURE 11: (a) Healthy aluminum sheet and (b) aluminum sheet with damage.

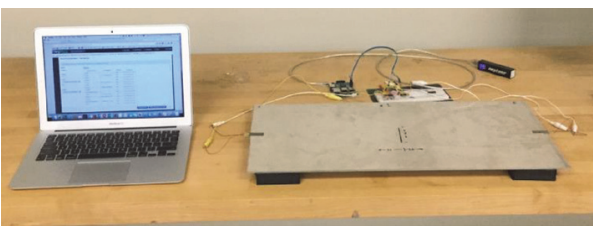

FIGURE 12: Test bed.

unhealthy sheet has damage that is $30 \mathrm{~cm}$ far from the exciter piezoelectric sensor and $7 \mathrm{~cm}$ width as shown in Figure 11. The proposed platform is used to check both sheets and send the data to the Internet server. These two sheets are tested separately by the proposed platform [45, 46]. Figure 12 shows the test bed as located in the lab. The health status was sent to the Internet server that hosted on ThingWorx [47]. ThingWorx is a technology platform designed for the 


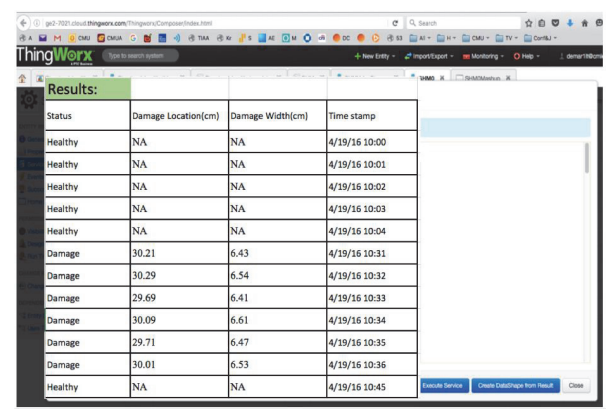

FIGURE 13: Results on the Internet server.

TABLE 1: Comparison between the data collected by the proposed platform and the actual sheet's status.

\begin{tabular}{|c|c|c|c|c|}
\hline \multirow[b]{2}{*}{$\begin{array}{l}\text { Actual } \\
\text { status }\end{array}$} & \multicolumn{4}{|c|}{ Results on the Internet } \\
\hline & $\begin{array}{l}\text { Damage } \\
\text { location } \\
(\mathrm{cm})\end{array}$ & $\begin{array}{c}\text { Damage } \\
\text { width } \\
(\mathrm{cm})\end{array}$ & $\begin{array}{c}\% \text { error of } \\
\text { the } \\
\text { damage } \\
\text { location }\end{array}$ & $\begin{array}{c}\% \text { error of } \\
\text { the } \\
\text { damage } \\
\text { width }\end{array}$ \\
\hline Healthy & NA & NA & NA & NA \\
\hline Healthy & NA & NA & NA & NA \\
\hline Healthy & NA & NA & NA & NA \\
\hline Healthy & NA & NA & NA & NA \\
\hline Healthy & NA & NA & NA & NA \\
\hline Damage & 30.21 & 6.43 & $0.7 \%$ & $8.14 \%$ \\
\hline Damage & 30.29 & 6.54 & $0.97 \%$ & $6.57 \%$ \\
\hline Damage & 29.69 & 6.41 & $1.03 \%$ & $8.43 \%$ \\
\hline Damage & 30.09 & 6.61 & $0.3 \%$ & $5.57 \%$ \\
\hline Damage & 29.71 & 6.47 & $0.97 \%$ & $7.57 \%$ \\
\hline Damage & 30.01 & 6.53 & $0.03 \%$ & $6.71 \%$ \\
\hline Healthy & NA & NA & NA & NA \\
\hline Healthy & NA & NA & NA & NA \\
\hline
\end{tabular}

IoT framework. It simplifies the creation and deployment of applications for smart, connected products by giving developers the tools they need to connect, build, analyze, experience, and collaborate about their "things." Figure 13 shows the results on ThingWorx.

Table 1 shows the percentage error of both the damage location and damage width between the actual sheets status and the data on the Internet. Results show that the proposed IoT SHM platform successfully checked if the sheet is healthy or not with $0 \%$ error. In addition, the proposed platform has a maximum of $1.03 \%$ error for the damage location and a maximum of $8.43 \%$ error for the damage width.

\section{Conclusion}

In this paper, a complete real-time IoT platform for SHM was proposed. The proposed platform consists of a Wi-Fi module, Raspberry Pi, DAC, ADC, buffer, and PZTs. The two PZTs are mounted on the structure and connected to a high speed ADC. A buffer was used as a level conversion and to protect the Raspberry Pi. The Raspberry Pi generates the excitation signal and the DAC converts it to analog. In addition, the Raspberry Pi was used to detect if the structure has damage or not. Moreover, the Raspberry was used to send the structure health status to the Internet server. The data was stored on the Internet server and can be monitored remotely from any mobile device. The system has been validated using a real test bed in the lab. Results show that the proposed IoT SHM platform successfully checked if the sheet is healthy or not with $0 \%$ error. In addition, the proposed platform has a maximum of $1.03 \%$ error for the damage location and a maximum of $8.43 \%$ error for the damage width.

\section{Competing Interests}

The authors declare that they have no competing interests.

\section{Acknowledgments}

The authors would like to thank the Office of Research and Sponsored Programs (ORSP) at Central Michigan University (CMU) for the valuable support (Grant no. 42487).

\section{References}

[1] X. Hu, B. Wang, and H. Ji, "A wireless sensor network-based structural health monitoring system for highway bridges," Computer-Aided Civil and Infrastructure Engineering, vol. 28, no. 3, pp. 193-209, 2013.

[2] S. Kim, S. Pakzad, D. Culler et al., Health Monitoring of Civil Infrastructures Using Wireless Sensor Networks, EECS Department, University of California, 2006.

[3] Z.-F. Gao, Y.-L. Du, M.-B. Su, and B. Chen, "Network sensor and its application in structure health monitoring system," in Proceedings of the 1st International Conference on Innovative Computing, Information and Control (ICICIC '06), pp. 68-71, August-September 2006.

[4] W. J. Staszewski, B. C. Lee, L. Mallet, and F. Scarpa, "Structural health monitoring using scanning laser vibrometry: I. Lamb wave sensing," Smart Materials and Structures, vol. 13, no. 2, pp. 251-260, 2004.

[5] W. J. Staszewski, B. C. Lee, and R. Traynor, "Fatigue crack detection in metallic structures with Lamb waves and 3D laser vibrometry," Measurement Science and Technology, vol. 18, no. 3, pp. 727-739, 2007.

[6] H. S. Park, H. M. Lee, H. Adeli, and I. Lee, "A new approach for health monitoring of structures: terrestrial laser scanning," Computer-Aided Civil and Infrastructure Engineering, vol. 22, no. 1, pp. 19-30, 2007.

[7] J. S. Hall and J. E. Michaels, "Multipath ultrasonic guided wave imaging in complex structures," Structural Health Monitoring, vol. 14, no. 4, pp. 345-358, 2015.

[8] D. Dai and Q. He, "Structure damage localization with ultrasonic guided waves based on a time-frequency method," Signal Processing, vol. 96, pp. 21-28, 2014.

[9] A. Basharat, N. Catbas, and M. Shah, "A framework for intelligent sensor network with video camera for structural health monitoring of bridges," in Proceedings of the 3rd IEEE International Conference on Pervasive Computing and Communications Workshops (PerCom Workshops '05), pp. 385-389, Kauai Island, Hawaii, USA, March 2005. 
[10] H. Sohn, D. Dutta, J. Y. Yang et al., "Delamination detection in composites through guided wave field image processing," Composites Science and Technology, vol. 71, no. 9, pp. 1250-1256, 2011.

[11] D. Liang, L. Wu, Z. Fan, and Y. Xu, "Self-diagnosis and selfreconfiguration of piezoelectric actuator and sensor network for large structural health monitoring," International Journal of Distributed Sensor Networks, vol. 11, no. 4, Article ID 207303, 2015.

[12] J. R. Wait, G. Park, and C. R. Farrar, "Integrated structural health assessment using piezoelectric active sensors," Shock and Vibration, vol. 12, no. 6, pp. 389-405, 2005.

[13] V. Giurgiuțiu, "Structural health monitoring with piezoelectric wafer active sensors-predictive modeling and simulation," INCAS Bulletin, vol. 2, no. 3, pp. 31-44, 2010.

[14] L. Yu and V. Giurgiutiu, "Piezoelectric wafer active sensors in lamb wave-based structural health monitoring," JOM, vol. 64, no. 7, pp. 814-822, 2012.

[15] E. F. Crawley and J. De Luis, "Use of piezoelectric actuators as elements of intelligent structures," AIAA Journal, vol. 25, no. 10, pp. 1373-1385, 1987.

[16] V. T. Rathod and D. R. Mahapatra, "Lamb wave based monitoring of plate-stiffener deboding using a circular array of piezoelectric sensors," International Journal on Smart Sensing and Intelligent Systems, vol. 3, no. 1, pp. 27-44, 2010.

[17] V. Giurgiutiu, Structural Health Monitoring: with Piezoelectric Wafer Active Sensors, Academic Press, 2007.

[18] H. Boukabache, C. Escriba, S. Zedek et al., "Structural health monitoring on metallic aircrafts using flexible and bulk pzt transducers: case of corrosion detection and crack localization," Stress, vol. 10, p. 2, 2012.

[19] V. Giurgiutiu, "Piezoelectric wafer active sensors for structural health monitoring of composite structures using tuned guided waves," Journal of Engineering Materials and Technology, vol. 133, no. 4, Article ID 041012, 2011.

[20] G. B. Santoni, L. Yu, B. Xu, and V. Giurgiutiu, "Lamb wave-mode tuning of piezoelectric wafer active sensors for structural health monitoring," Journal of Vibration and Acoustics, vol. 129, no. 6, pp. 752-762, 2007.

[21] V. Giurgiutiu and G. Santoni-Bottai, "Structural health monitoring of composite structures with piezoelectric-wafer active sensors," AIAA Journal, vol. 49, no. 3, pp. 565-581, 2011.

[22] R. C. Tennyson, A. A. Mufti, S. Rizkalla, G. Tadros, and B. Benmokrane, "Structural health monitoring of innovative bridges in Canada with fiber optic sensors," Smart Materials and Structures, vol. 10, no. 3, pp. 560-573, 2001.

[23] J. P. Amezquita-Sanchez and H. Adeli, "Signal processing techniques for vibration-based health monitoring of smart structures," Archives of Computational Methods in Engineering, vol. 23, no. 1, pp. 1-15, 2016.

[24] G. Hackmann, W. Guo, G. Yan, Z. Sun, C. Lu, and S. Dyke, "Cyber-physical codesign of distributed structural health monitoring with wireless sensor networks," IEEE Transactions on Parallel and Distributed Systems, vol. 25, no. 1, pp. 63-72, 2014.

[25] J. Paek, N. Kothari, K. Chintalapudi et al., The Performance of a Wireless Sensor Network for Structural Health Monitoring, Center for Embedded Network Sensing, 2004.

[26] H. Zhang, J. Guo, X. Xie, R. Bie, and Y. Sun, "Environmental effect removal based structural health monitoring in the internet of things," in Proceedings of the 7th International Conference on Innovative Mobile and Internet Services in Ubiquitous Computing (IMIS '13), pp. 512-517, July 2013.
[27] L.-S. Huo, X. Li, Y.-B. Yang, and H.-N. Li, "Damage detection of structures for ambient loading based on cross correlation function amplitude and SVM," Shock and Vibration, vol. 2016, Article ID 3989743, 12 pages, 2016.

[28] S. Park, S. R. Anton, J.-K. Kim, D. J. Inman, and D. S. Ha, "Instantaneous baseline structural damage detection using a miniaturized piezoelectric guided waves system," KSCE Journal of Civil Engineering, vol. 14, no. 6, pp. 889-895, 2010.

[29] S. Gao, X. Dai, Z. Liu, and G. Tian, "High-performance wireless piezoelectric sensor network for distributed structural health monitoring," International Journal of Distributed Sensor Networks, vol. 2016, Article ID 3846804, 16 pages, 2016.

[30] D. Samaratunga, R. Jha, and S. Gopalakrishnan, "Wave propagation analysis in laminated composite plates with transverse cracks using the wavelet spectral finite element method," Finite Elements in Analysis and Design, vol. 89, pp. 19-32, 2014.

[31] A. Hera and Z. Hou, "Application of wavelet approach for ASCE structural health monitoring benchmark studies," Journal of Engineering Mechanics, vol. 130, no. 1, pp. 96-104, 2004.

[32] X. Liu, J. Cao, W.-Z. Song, P. Guo, and Z. He, "Distributed sensing for high-quality structural health monitoring using WSNs," IEEE Transactions on Parallel and Distributed Systems, vol. 26, no. 3, pp. 738-747, 2015.

[33] M. Bocca, J. Toivola, L. M. Eriksson, J. Hollmén, and H. Koivo, "Structural health monitoring in wireless sensor networks by the embedded goertzel algorithm," in Proceedings of the IEEE/ACM 2nd International Conference on Cyber-Physical Systems (ICCPS '11), pp. 206-214, April 2011.

[34] M. Z. A. Bhuiyan, G. Wang, J. Cao, and J. Wu, "Deploying wireless sensor networks with fault-tolerance for structural health monitoring," IEEE Transactions on Computers, vol. 64, no. 2, pp. 382-395, 2015.

[35] Y. Bao, Y. Yu, H. Li et al., "Compressive sensing-based lost data recovery of fast-moving wireless sensing for structural health monitoring," Structural Control and Health Monitoring, vol. 22, no. 3, pp. 433-448, 2014.

[36] C. Lazo, P. Gallardo, and S. Céspedes, "A bridge structural health monitoring system supported by the Internet of Things," in Proceedings of the IEEE Colombian Conference on Communications and Computing (COLCOM '15), pp. 1-6, Popayán, Colombia, May 2015.

[37] J. P. Lynch and K. J. Loh, "A summary review of wireless sensors and sensor networks for structural health monitoring," Shock and Vibration Digest, vol. 38, no. 2, pp. 91-130, 2006.

[38] I. Kim and A. Chattopadhyay, "Guided Lamb wave-based structural health monitoring using a novel wave packet tracing method for damage localization and size quantification," Journal of Intelligent Material Systems and Structures, vol. 26, no. 18, pp. 2515-2530, 2015.

[39] ABAQUS software website, 2016, http://buyabaqus.com/.

[40] Mtlab software website, 2016, http://www.mathworks.com/.

[41] A. Abdelgawad and K. Yelamarthi, "Structural health monitoring: internet of things application," in Proceedings of the 59th IEEE International Midwest Symposium on Circuits and Systems (MWSCAS '16), Abu Dhabi, United Arab Emirates, October 2016.

[42] Intersil, “CA3306 ADC Data Sheet," 2016, http://media.digikey .com/pdf/Data\%20Sheets/Intersil\%20PDFs/CA3306\%28A,C\% 29.pdf.

[43] MCP4725 Datasheet, 2016, https://cdn-shop.adafruit.com/datasheets/mcp4725.pdf. 
[44] 74HC4050 Datasheet, 2016, http://www.nxp.com/documents/ data_sheet/74HC4050.pdf.

[45] A. Myers, A. Mahmud, A. Abdelgawad, and K. Yelamarthi, "Toward integrating structural health monitoring with internet of things," in Proceedings of the IEEE International Conference on Electro/Information Technology, May 2016.

[46] K. Laubhan, K. Talaat, S. Riehl, T. Morelli, A. Abdelgawad, and K. Yelamarthi, "A four-layer wireless sensor network framework for IoT applications," in Proceedings of the 59th IEEE International Midwest Symposium on Circuits and Systems (MWSCAS '16), October 2016.

[47] "Thingworx website [Online]," June 2016, http://www.thingworx.com. 


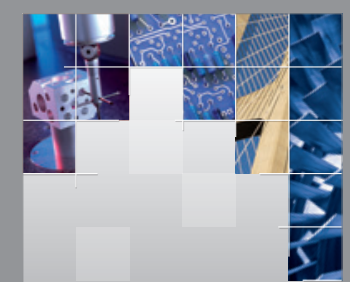

\section{Enfincering}
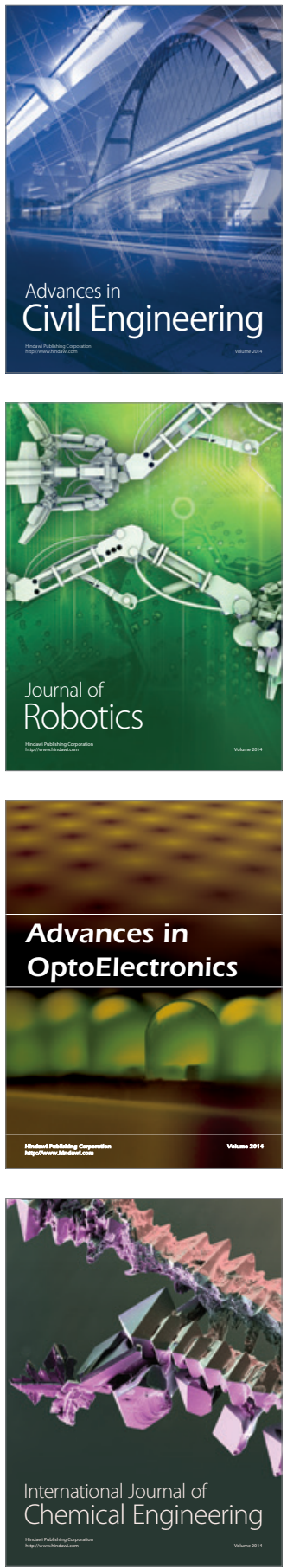

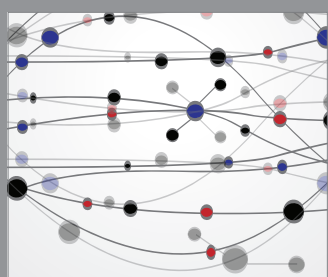

The Scientific World Journal

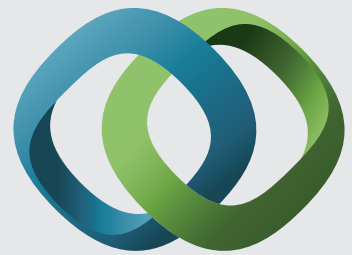

\section{Hindawi}

Submit your manuscripts at

https://www.hindawi.com
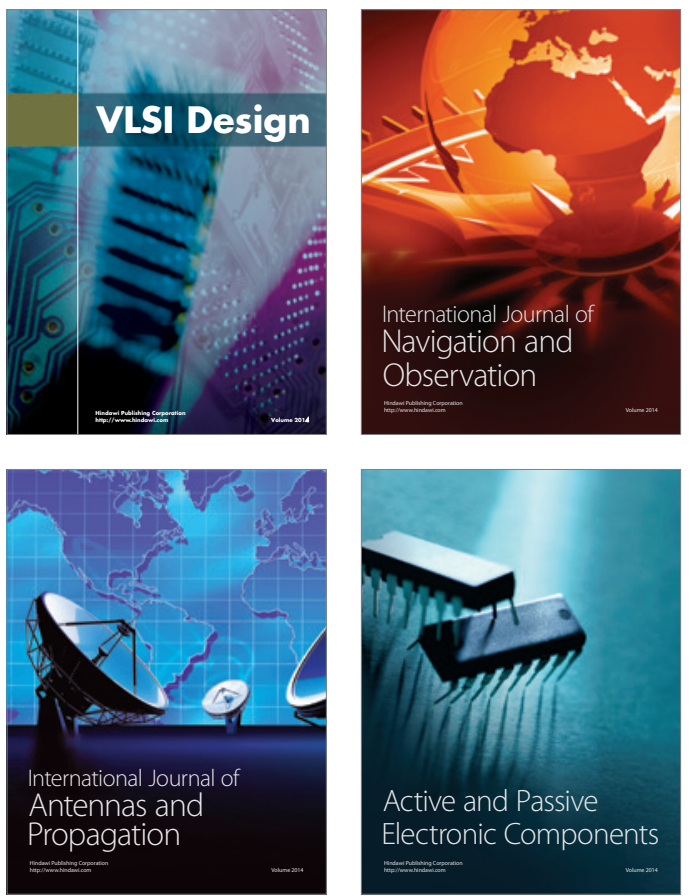
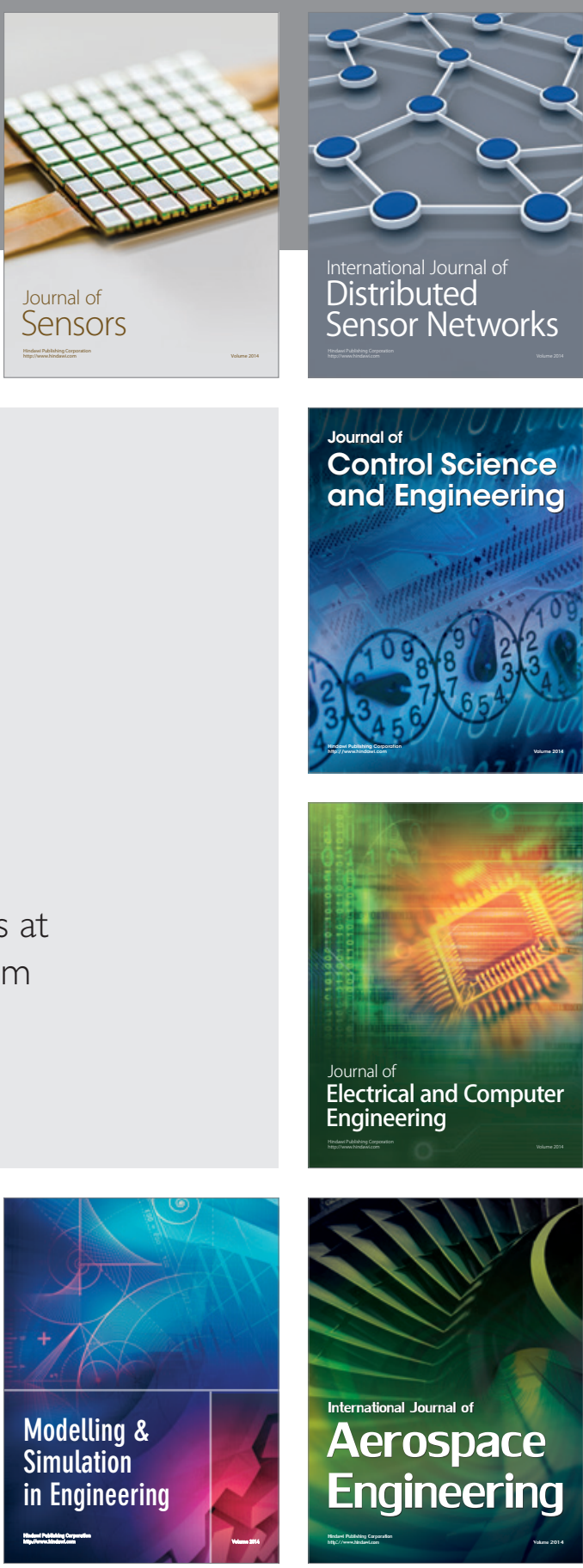

International Journal of

Distributed

Sensor Networks

$-$

Joumal of

Control Science

and Engineering
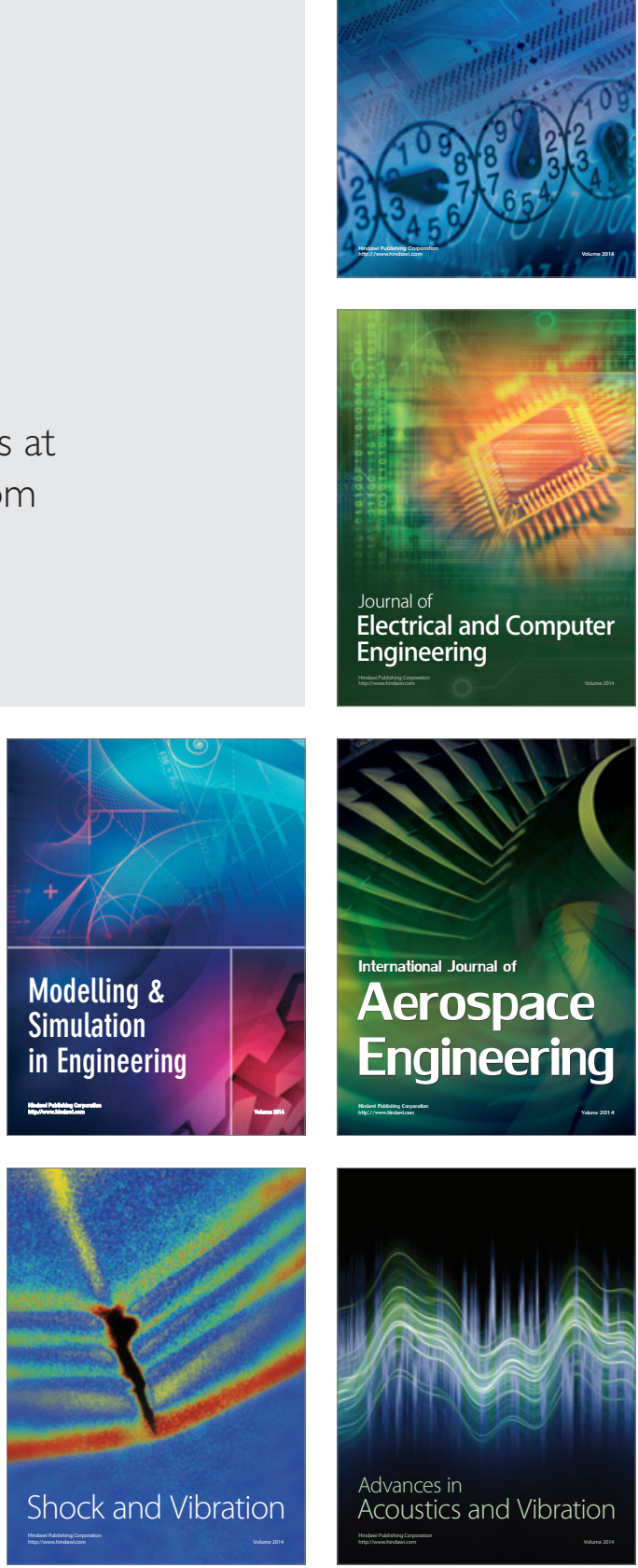